\title{
Retinal nerve fiber layer and ganglion cell layer changes using optical coherence tomography in patients with chronic migraine: a case-control study
}

\author{
Dalia M. Labib ${ }^{1 *}$, Montaser Hegazy ${ }^{1}$, Soheir Mohamed Esmat ${ }^{2}$, Enas Abdel Hamid Ali ${ }^{3}$ and Foraysa Talaat ${ }^{1}$
}

\begin{abstract}
Background: Migraine is a prevalent, chronic, and multifactorial neurovascular disease.

Objectives: Our work aimed to investigate if the retinal nerve fiber layer (RNFL) and ganglion cell layer (GCL) thickness are affected in patients with chronic migraine to improve the understanding of the etiology and pathophysiology of migraine.
\end{abstract}

Subjects and methods: A case-control study conducted on 30 patients with chronic migraine and 30 aged and sexmatched healthy controls. Subjects underwent full neurological and ophthalmological history, ophthalmological examination, and measuring RNFL and GCL thickness using the spectral domain-optical coherence tomography (SD-OCT).

Results: RNFL thinning (average, superior, inferior, nasal, and temporal) was significantly more in patients with chronic migraine than healthy control $(P=0.001,0.022,0.045,0.034$, and 0.001 , respectively). No statistically significant difference was found between chronic migraine patients and healthy controls regarding GCL thickness (average, superior, and inferior) ( $P$ value > 0.05).

The average RNFL thickness was significantly thinner in migraine with aura (MwA) than migraine without aura (MwoA) $(P$ $=0.006)$. The average $G C L$ thickness was thinner in MwA than MwoA $(P=0.039)$. No statistically significant difference was found between the eyes on the side of the headache and the eyes of the contralateral side regarding RNFL and GCL thickness ( $P$ value > 0.05). Age at onset, disease duration, headache frequency, and headache intensity showed an insignificant correlation with OCT parameters.

Conclusion: Retinal changes could be an association with chronic migraine that may be used as a biomarker.

Keywords: Chronic migraine, Optical coherence tomography, Ganglion cell complex, Retinal nerve fiber layer

\section{Introduction}

Migraine is a highly prevalent neurological disease that affects about $15 \%$ of the general population [1]. It is the sixth highest cause of disability worldwide [2]. It is characterized by moderate to severe recurrent episodes of unilateral, throbbing, or pulsating headache that may be associated with nausea, vomiting, phonophobia, and/or

\footnotetext{
* Correspondence: dr.dalia.neuro@gmail.com

${ }^{1}$ Neurology Department, Faculty of Medicine, Cairo University, Cairo, Egypt Full list of author information is available at the end of the article
}

photophobia [3, 4]. Clinically, migraine is divided into two main subtypes: migraine with aura (MwA or classic migraine) and migraine without aura (MwoA or common migraine) [5]. It is estimated that MwA affects 10 to $30 \%$ of migraine patients; the aura symptoms appear shortly before or during the development of migraine headaches or no headache may follow [6]. Aura develops over 5 to 20 min and lasts less than an hour. The most common aura symptoms involve the vision, with hallucination/ illusion of bright flashing lights and temporary blindness. 
Chronic migraine is one of the chronic daily headaches. It is defined as more than fifteen headache days per month over 3 months of which more than eight are migrainous, in the absence of medication overuse, whereas episodic migraine is defined as up to fourteen headache days per month. Chronic migraine affects less than $1 \%$ of the population [7]. Its impact can be disabling and the World Health Organization has categorized it as the same disability as dementia and quadriplegia and it is more disabling than blindness and paraplegia [8].

Despite the underlying pathophysiology of migraine is not fully established and many theories have been suggested [9], the neurovascular theory remains one of the most significant mechanisms involved in the pathogenesis of migraine. Neural, vascular, and inflammatory events in migraine headaches are initiated by the activation and sensitization of the trigeminovascular system (TGVS) $[10,11]$ which consists of the trigeminal nerve fibers that innervate the extracranial and intracranial meningeal blood vessels and ocular structures [12]. The activation of the TGVS inspires the release of vasoactive neurotransmitters from peripheral endings of the trigeminal nerve, resulting in the associated vascular and inflammatory changes that causing pain $[10,13]$. Although the temporary cerebral vasospasm that occurs before or during pain leads to a reduction in cerebral blood flow which is limited to the posterior part of one hemisphere, it ends in cerebral hypoperfusion which may involve other areas outside the brain, such as the retina [14]. Despite the arterial vasospasm is a transient phenomenon, the chronic nature of the migraine may lead to permanent structural changes in the brain and retina as permanent ganglion cell damage [14, 15].

Furthermore, migraine is a well-known risk factor for ischemic optic neuropathy and normal-tension glaucoma (NTG) because of the retinal ischemia which arises from retinal artery occlusion [15].

The anatomical structure of the retina is considered to be an extension of the brain. The retinal nerve fiber layer (RNFL) is similar to the gray matter of the brain, and its thickness changes are simply due to axonal damage. From this point of view, the retina is considered to be an easily accessible window into the brain.

Optical coherence tomography (OCT) is a noninvasive imaging procedure that gives high-resolution, crosssectional images of the RNFL, ganglion cell layer (GCL), and the optic nerve head [16].

Our study aimed to investigate if the RNFL thickness and GCL thickness are affected in patients with chronic migraine to improve the understanding of the etiology and pathophysiology of migraine.

\section{Subjects and methods}

This cross-sectional case-control study was conducted on 30 Egyptian patients with chronic migraine [fifteen with aura (MwA), and fifteen without aura (MwoA)]. All the patients met the migraine diagnostic criteria of the headache classification committee of the international headache society, 3rd edition (beta version) [5]. Patients were recruited from headache outpatient clinic of Kasr Al- Ainy Hospitals, Cairo University, from August 2018 to February 2019.

We excluded patients who are smokers, diabetic, hypertensive, dyslipidemic, with chronic renal, hepatic disease, or cardiovascular disease, patients with a history of collagen vascular diseases. Also, patients with a history of central nervous system disorders such as brain tumors, infarction, multiple sclerosis, patients with glaucoma, eye trauma, diabetic neuropathy, dense cataract, corneal opacity, uncorrected refractive error, and history of ocular surgery were excluded.

Thirty healthy controls, with no history of ocular or neurological disease, were recruited from the family members of the patients.

The study was approved by the local ethical committee of the Faculty of Medicine, Cairo University (according to the WMA Declaration of Helsinki), and all the participants gave their informed consent.

The patients were subjected to full neurological history and examination with special emphasis on disease duration, frequency of migraine (attacks per month), duration of migraine headache with and without medications, the severity of the migraine attack (using Numeric Pain Rating Scale) [17], location, character of migraine headache and relation to sleep, associated symptoms, aura symptoms, precipitating and aggravating, and relieving factors.

Full ophthalmological examination of each eye including best-corrected visual acuity (BCVA) testing using Snellen's charts, visual fields by confrontation, slit lamp examination, intraocular pressure measurement with tonometry, and fundus examination was performed for all participants.

Spectral-domain optical coherence tomography was performed using the Optovue RTVue XR Avanti ${ }^{\mathrm{m}}$ (Optovu, inc. Fremont, CA, USA) at the laser unit of the Kasr Alainy Hospitals. The optic disk map was used to estimate the average thickness of peripapillary RNFL and the thickness in the superior, inferior, nasal, and temporal quadrants. The macular map protocol was used to measure the GCL thickness, and it was divided into superior and inferior hemispheres. All scans were carried out with ambient lighting and without pupil dilation to ensure patient comfort. Both eyes were examined in all participants and the procedure was completed in about $10 \mathrm{~min}$.

\section{Statistical analysis}

Statistical calculations were done using computer programs SPSS (Statistical Package for the Social Science; 
SPSS Inc., Chicago, IL, USA) version 19 for Microsoft Windows. Data were expressed as mean \pm stander deviation (SD) for the parametric variables and as number and percent for the non-parametric variable. A comparison between groups for parametric data was done by independent samples $t$ test (unpaired $t$ test). Chi-square $\left(X^{2}\right)$ test was used to compare qualitative variables. Pearson and Spearman correlation coefficients $(r)$ were calculated for the detection of parametric and nonparametric correlations, respectively. The difference was expressed as a probability of value ( $P$ value). The difference was considered significant if $P<0.05$.

\section{Results}

Thirty migraineurs were included in the study; 25 women, 5 men, mean age $30.6 \pm 6.7$ years, range $18-49$ years; mean disease duration $9.23 \pm 5.12$ years, range 2 20 years; the mean number of attacks per month $9.4 \pm$ 2.465 , range $5-14$. The duration of the migraine attack without acute medications ranged from 4 to $72 \mathrm{~h}$ with a mean of $30.53 \pm 18.47$.

Fifteen patients had a migraine with aura (50\%); 12 patients had visual aura (80\%); 2 patients (13\%) had mixed auras and visual and sensory auras; and 1 patient had retinal aura (7\%).

Regarding migraine headache intensity, the Numeric Rating Scale (NRS) ranged from 5 to 10 with a mean of $7.37 \pm 1.089$.

Eight patients (26.7\%) had their migraine headache attacks on the right side. Seven patients $(23.3 \%)$ had their migraine headache attacks on the left side while fifteen patients $(50 \%)$ had their migraine headache attacks on both sides.

RNFL was significantly thinner in patients compared to healthy controls while no significant difference was found between the GCL layer of patients and healthy controls, as shown in Table 1.

RNFL was significantly thinner in the inferior and nasal quadrants in the patients with MwA than the patients with MwoA, and there was a significant GCL thinning of the superior half in patients with MwA, as shown in Table 2.

RNFL and GCL thickness were not statistically different between the eyes on the side of the headache and the eyes on the contralateral side, as shown in Table 3.

No significant correlation was detected between OCT parameters with age, headache duration, headache frequency, and headache intensity, as shown in Table 4.

\section{Discussion}

Our study aimed to investigate if the RNFL thickness and GCL thickness are affected in patients with a chronic migraine that may improve the understanding of the etiology and pathophysiology of migraine.

In our study, the average RNFL thickness and the RNFL thickness of all quadrants (superior, inferior, nasal, and temporal) were significantly thinner in the chronic migraine patients, either with or without aura, than the healthy controls. The diminished RNFL thickness means a reduction in the number of axons in migraine patients. Our results come in agreement with different previous studies [18-20]. Other studies have demonstrated only RNFL thinning in a specific quadrant; Colak and colleagues [21] reported RNFL thinning in the superior and inferior quadrants, while Martinez and colleagues [22] reported significant decreased RNFL thickness in the temporal quadrant only. Some studies have observed that RNFL thickness was thin in the nasal quadrant only $[23,24]$ and others have reported RNFL thinning in the superior quadrant $[25,26]$. The selective RNFL involvement was attributed to the differences in the vulnerability of the axons to retinal ischemia [27].

On the other hand, Simsek and coworkers [14] reported that no significant difference in the average RNFL thickness or any of the quadrants in migraine patients, with or without aura, and healthy controls except for the nasal quadrant of the right eye, which had a significantly higher value. Also, two previous studies found $[16,28]$

Table 1 Comparison of OCT parameters between patients with migraine and healthy controls

\begin{tabular}{llll}
\hline OCT parameters & $\begin{array}{l}\text { Migraine patients, } \boldsymbol{N}=(60 \text { eyes }) \\
\text { Mean } \pm \text { SD }\end{array}$ & $\begin{array}{l}\text { Healthy controls, } \boldsymbol{N}=(60 \text { eyes }) \\
\text { Mean } \pm \text { SD }\end{array}$ \\
\hline AVR-RNFL $(\mathbf{u m})$ & $103.7 \pm 7.157$ & $112.85 \pm 5.960$ & $<0.001^{*}$ \\
SUP-RNFL $(\boldsymbol{\mu m})$ & $125.75 \pm 10.088$ & $131.35 \pm 19.673$ & $0.022^{*}$ \\
INF-RNFL $(\boldsymbol{\mu m})$ & $129.68 \pm 13.363$ & $136.20 \pm 19.230$ & $0.045^{*}$ \\
NAS-RNFL $(\boldsymbol{\mu m})$ & $80.27 \pm 8.481$ & $90.48 \pm 6.008$ & $0.034^{*}$ \\
TEMP-RNFL $(\boldsymbol{\mu m})$ & $77.68 \pm 8.584$ & $93.38 \pm 7.863$ & $<0.001^{*}$ \\
AVR-GCL $(\boldsymbol{\mu m})$ & $98.95 \pm 5.350$ & $100.52 \pm 8.955$ & 0.247 \\
SUP-GCL $(\boldsymbol{\mu m})$ & $98.65 \pm 5.065$ & $98.65 \pm 8.346$ & 1.000 \\
INF-GCL $(\boldsymbol{\mu m})$ & $99.17 \pm 5.708$ & $97.28 \pm 7.355$ & 0.119 \\
\hline AVR
\end{tabular}

AVR average, GCL ganglion cell layer, INF inferior, NAS nasal, OCT optical coherence tomography, RNFL retinal nerve fiber layer, SUP superior, TEMP temporal *Significant $P$ value $<0.05$ 
Table 2 Comparison of the OCT parameters between patients with MwA and MwoA

\begin{tabular}{llll}
\hline OCT parameters & MwA, $\boldsymbol{N}=(30$ eyes $)$ & $\begin{array}{l}\text { MwoA, } \boldsymbol{N}=(30 \text { eyes }) \\
\end{array}$ & $\begin{array}{l}\boldsymbol{P} \\
\text { value }\end{array}$ \\
\hline AVR-RNFL $(\mathbf{u m})$ & $101.23 \pm 6.468$ & $106.20 \pm 7.044$ & $0.006^{*}$ \\
SUP-RNFL $(\boldsymbol{\mu m})$ & $123.37 \pm 9.205$ & $127.77 \pm 10.598$ & 0.091 \\
INF-RNFL $(\boldsymbol{\mu m})$ & $125.80 \pm 13.045$ & $133.57 \pm 13.302$ & $0.026^{*}$ \\
NAS-RNFL $(\boldsymbol{\mu m})$ & $78.07 \pm 6.395$ & $82.47 \pm 9.769$ & $0.043^{*}$ \\
TEMP-RNFL( $\boldsymbol{\mu m})$ & $76.03 \pm 7.618$ & $79.33 \pm 9.286$ & 0.138 \\
AVR-GCL $(\boldsymbol{\mu m})$ & $97.53 \pm 5.224$ & $100.37 \pm 5.176$ & $0.039^{*}$ \\
SUP-GCL( $\boldsymbol{\mu m})$ & $96.93 \pm 4.899$ & $100.37 \pm 4.701$ & $0.008^{*}$ \\
INF-GCL( $\boldsymbol{\mu m})$ & $98.27 \pm 5.723$ & $100.07 \pm 5.644$ & 0.225 \\
\hline AVR
\end{tabular}

$A V R$ average, GCL ganglion cell layer, INF inferior, MwA migraine with aura, MWoA migraine without aura, NAS nasal, OCT optical coherence tomography, $R N F L$ retinal nerve fiber layer, SUP superior, TEMP temporal

*Significant $P$ value 0.05

no statistically significant differences in the retinal thickness between migraine patients and healthy control were found. Finding no significant difference in RNFL may be due to short mean disease duration or low numbers of migraine attacks in those studies [16].

Regarding the GCL thickness, in our study, we found no statistically significant difference between the GCL thickness in migraine patients and healthy control. This goes in agreement with a few earlier reports [21, 29]. However, several studies showed the opposite of what we found [16, 19, 30]; Abdellatif and Fouad [15] reported that the superior and inferior GCL thicknesses were significantly diminished in patients with migraine, either MwA or MwoA, compared to healthy controls. Reggio and colleagues [19] hypothesized that the alteration in the blood supply to the anterior optic nerve head results in an oligemic-hypoxic insult that contributes to ganglion cell damage [19].

Table 3 Comparison between the OCT parameters in the ipsilateral eyes and the contralateral eyes

\begin{tabular}{llll}
\hline OCT parameters & $\begin{array}{l}\text { Ipsilateral eye } \\
\text { (15 eyes) } \\
\text { Mean } \pm \text { SD }\end{array}$ & $\begin{array}{l}\text { Contralateral eye } \\
\text { (15 eyes) } \\
\text { Mean } \pm \text { SD }\end{array}$ & $P$ value \\
\hline AVR- RNFL $(\mathbf{u m})$ & $104.27 \pm 5.934$ & $105.40 \pm 6.780$ & 0.873 \\
SUP- RNFL $(\boldsymbol{\mu m})$ & $126.67 \pm 8.780$ & $129.20 \pm 7.966$ & 0.877 \\
INF- RNFL $(\boldsymbol{\mu m})$ & $126.93 \pm 13.714$ & $130.13 \pm 11.825$ & 0.867 \\
NAS-RNFL $(\boldsymbol{\mu m})$ & $80.60 \pm 10.190$ & $81.93 \pm 8.075$ & 0.888 \\
TEMP-RNFL $(\boldsymbol{\mu m})$ & $78.07 \pm 11.310$ & $79.87 \pm 8.935$ & 0.853 \\
AVR- GCL $(\boldsymbol{\mu m})$ & $100.46 \pm 6.13$ & $99.93 \pm 5.70$ & 0.792 \\
SUP- GCL $(\boldsymbol{\mu m})$ & $100.33 \pm 5.67$ & $99.93 \pm 5.18$ & 0.759 \\
INF- GCL $(\boldsymbol{\mu m})$ & $100.33 \pm 6.78$ & $99.73 \pm 6.07$ & 0.723 \\
\hline
\end{tabular}

AVR average, GCL ganglion cell layer, INF inferior, NAS nasal, OCT optical coherence tomography, RNFL retinal nerve fiber layer, SUP superior, TEMP temporal
Table 4 Correlation between OCT parameters with age, disease duration, headache frequency, and headache intensity

\begin{tabular}{llll}
\hline Variables & & Correlation coefficient & $\boldsymbol{P}$ value \\
\hline RNFL & Age & -0.138 & 0.295 \\
& Disease duration & -0.143 & 0.276 \\
& Frequency & 0.161 & 0.218 \\
& Intensity & -0.019 & 0.885 \\
\multirow{2}{*}{ GCL } & Age & -0.120 & 0.361 \\
& Disease duration & -0.219 & 0.092 \\
& Frequency & 0.210 & 0.108 \\
& Intensity & -0.113 & 0.389
\end{tabular}

GCL ganglion cell layer, OCT optical coherence tomography, RNFL retinal nerve fiber layer

We further analyzed the patients with migraine into subgroups: MwA and MwoA; in which we found a significant thinning of the average, inferior, and nasal quadrants of RNFL thickness in patients with MwA than patients with MwoA.

Our study results are in agreement with a study achieved by Ao and coworkers, who reported a significant reduction in the RNFL thickness of the nasal and inferior quadrants in patients with MwA compared to MwoA [24]. Also, the study of Tunç and colleagues showed a significant reduction in the RNFL thickness of the average and superior quadrants between MwA and MwoA [29]. The study of Ekinci and coworkers found non-significant more thinning of the RNFL in patients with MwA than patients with MwoA [30].

During the aura of migraine, the posterior part of the cerebral hemisphere shows cerebral hypoperfusion which can explain the more RNFL thinning in MwA compared to MwoA [22]. However, some studies found no significant difference in the RNFL thickness between patients with MwA and MwoA [14, 19, 31].

Regarding the GCL thickness between the migraine subgroups; MwA and MwoA, our study found that the GCL thickness was thinner in patients with MwA than in patients with MwoA. There was a statistically significant difference in the average and superior half GCL thickness between patients with MwA and patients with MwoA. Our results were in agreement with previous studies $[19,26,30]$. On the other hand, it was found no significant difference in the GCL thickness between patients with MwA and patients with MwoA [20,21].

As migraine patients experience headaches almost on the one side, we studied the ipsilateral involvement of the RNFL and we found no significant difference in RNFL thickness between the eyes on the side of the headache and the eyes on the contralateral side. Our finding goes in concordant with the study of Gunes and colleagues [27] which had investigated the association between laterality of migraine and RNFL thickness 
headache side, and they found more thinning of RNFL on the same side of the headache and the asymmetry was not statistically significant [27]. Such thinning of RNFL on the headache side could be attributed to the chronic course of migraine with periodic reduction of the blood flow on the ipsilateral hemisphere during the attacks that could lead to permanent cerebral and retinal damage [32].

In agreement with previous studies, our results showed no significant correlation between the average RNFL or the average GCL thicknesses and the duration of the migraine, the attack frequency, or the severity of the migraine $[14,16,26,27]$.

Theoretically, migraine with long disease duration, higher frequency of attacks, or severe attacks might cause more damage to the RNFL and GCL thickness. In such a manner, Abdellatif and Fouad found that the duration of migraine was negatively correlated with superior and inferior RNFL and GCL, while the severity of migraine showed a significant negative correlation with inferior and temporal RNFL and the superior and inferior GCL [20]. Also, Martinez and colleagues reported a similar negative correlation between the severity and duration of migraine and RNFL thickness [22].

The disparity between the results of the current study and previous studies may be attributed to differences in methodology and sample size, mean age of the population, ethnic variations, and the absence of standardized migraine characteristics, including length of migraine history, severity, and frequency of attacks.

\section{Conclusion}

Migraine with both types (MwA and MwoA) could reduce the RNFL and GCL thicknesses. The axonal loss requires oversight on testing the RNFL thickness in migraine patients. Further researches may deduct the importance of OCT findings as an investigative surrogate for migraine.

\section{Abbreviations}

AVR: Average; BCVA: Best-corrected visual acuity; GCL: Ganglion cell layer; INF: Inferior; MwA: Migraine with aura; MwoA: Migraine without aura; NAS: Nasal; NRS: Numeric Rating Scale; NTG: Normal-tension glaucoma; OCT: Optical coherence tomography; $r$ : Correlation coefficients; RNFL: Retinal nerve fiber layer; SD: Stander deviation; SD-OCT: Spectral domain-optical coherence tomography; SPSS: Statistical Package for the Social Science; SUP: Superior; TEMP: Temporal; TGVS: Trigeminovascular system

\section{Acknowledgements}

Not applicable.

\section{Authors' contributions}

FT contributed to the research idea, data acquisition, data analysis, interpretation, and manuscript reviewing. $\mathrm{MH}$ contributed to data acquisition, data analysis, and interpretation. SME contributed to the OCT performance, data analysis, and interpretation. EAHA contributed to the data acquisition and data analysis and interpretation. DML contributed to the scientific writing of the manuscript, and she is responsible for the publication. The authors read and approved the final manuscript.

\section{Funding}

No funding was obtained from any institution for our study.

\section{Availability of data and materials}

The datasets generated and/or analyzed during the current study are not publicly available due to the current Cairo University regulations and Egyptian legislation, but they are available by a reasonable request from the corresponding author.

\section{Ethics approval and consent to participate}

All procedures performed in the study were in accordance with the ethical standards of the institutional research committee and with the 1964 Helsinki Declaration and its later amendments. Our study was approved by the ethical committee of the Department of Neurology, Faculty of Medicine, Cairo University, in June 2016, but Cairo University does not provide an approval reference number.

An informed written consent was taken from each participant involved in this study prior to the conduct of any study-related activities.

All data obtained from participants were confidential and were not used outside the study. The patients had the rights to withdraw from the study at any time without giving any reason.

\section{Consent for publication}

Not applicable

\section{Competing interests}

None of the authors has any conflict of interests.

\section{Author details}

${ }^{1}$ Neurology Department, Faculty of Medicine, Cairo University, Cairo, Egypt. ${ }^{2}$ Ophthalmology Department, Faculty of Medicine, Cairo University, Cairo, Egypt. ${ }^{3}$ Neurology Department, Nasser Institute Hospital, Cairo, Egypt.

Received: 14 April 2020 Accepted: 5 August 2020

Published online: 14 August 2020

\section{References}

1. Burch RC, Loder S, Loder E, Smitherman TA. The prevalence and burden of migraine and severe headache in the United States: updated statistics from government health surveillance studies. Headache. 2015;55(1):21.

2. Saylor D, Steiner TJ. The global burden of headache. Semin Neurol. 2018;38: 182.

3. Ascaso FJ, Marco S, Mateo J, Martínez M, Esteban O, Grzybowski A. Optical coherence tomography in patients with chronic migraine: literature review and update. Front Neurol. 2017;8:684

4. Global burden of disease study collaborators. Global, regional, and national incidence, prevalence, and years lived with disability for 301 acute and chronic diseases and injuries in 188 countries, 1990-2013: a systematic analysis for the Global Burden of Disease Study 2013. Lancet. 2015;386:743800.

5. Headache Classification Committee of the International Headache Society. The international classification of headache disorders, 3rd edition. Cephalalgia. 2018;38(1):1-211.

6. Vecchia D, Pietrobon D. Migraine: a disorder of brain excitatory-inhibitory balance? Trends Neurosci. 2012;35(8):507-20.

7. Olesen J, Bousser MG, Diener HC, Dodick D, First M, Goadsby PJ, et al. New appendix criteria open for a broader concept of chronic migraine. Cephalalgia. 2006;26:742-6.

8. Buse DC, Rupnow MF, Lipton RB. Assessing and managing all aspects of migraine: migraine attacks, migraine-related functional impairment, common comorbidities, and quality of life. Mayo Clin Proc. 2009;84:422-35.

9. Sacco S, Ricci S, Carolei A. Migraine and vascular diseases: a review of the evidence and potential implications for management. Cephalalgia. 2012;32: 785-95

10. Silberstein SD. Migraine pathophysiology and its clinical implications. Cephalalgia. 2004;24(Suppl. 2):2-7.

11. Ayata C. Cortical spreading depression triggers migraine attack: pro. Headache. 2010;50(4):725-30.

12. Aguggia M, Saracco MG, Cavallini M, Bussone G, Cortelli P. Sensitization and pain. Neurol Sci. 2013:34:S37-40.

13. Goadsby PJ. Pathophysiology of migraine. Neurol Clin. 2009;27(2):335-60. 
14. Simsek IB, Aygun D, Yildiz S. Retinal nerve fiber layer thickness in migraine patients with or without aura. Neuroophthalmology. 2015;39(1):17-21.

15. Flammer J, Pache M, Resink T. Vasospasm, its role in the pathogenesis of diseases with particular reference to the eye. Prog Retin Eye Res. 2001;20(3): 319-49.

16. Gunes A, Karadag AS, Yazgan S, Celik HU, Simsek A. Evaluation of retinal nerve fiber layer, ganglion cell layer and choroidal thickness with optical coherence tomography in migraine patients: a case-control study. Clin Exp Optom. 2018;101(1):109-15.

17. Jensen MP, Karoly P, O'Riordan EF, Bland F, Burns RS. The subjective experience of acute pain. An assessment of the utility of 10 indices. Clin J Pain. 1989;5:153-9.

18. Feng YF, Guo H, Huang JH, Yu JG, Yuan F. Retinal nerve fiber layer thickness changes in migraine: a meta-analysis of case-control studies. Curr Eye Res. 2016;41(6):814-22

19. Reggio E, Chisari CG, Ferrigno G, Patti F, Donzuso G, Sciacca G, et al. Migraine causes retinal and choroidal structural changes: evaluation with ocular coherence tomography. J Neurol. 2017;264(3):494-502.

20. Abdellatif MK, Fouad MM. Effect of duration and severity of migraine on retinal nerve fiber layer, ganglion cell layer, and choroidal thickness. Eur J Ophthalmol. 2018;28(6):714-21.

21. Colak HN, Kantarcı FA, Tatar MG, Eryilmaz M, Uslu H, Goker H, et al. Retinal nerve fiber layer, ganglion cell complex, and choroidal thicknesses in migraine. Arq Bras Oftalmol. 2016;79(2):78-81.

22. Martinez A, Proupim N, Sanchez M. Retinal nerve fiber layer thickness measurements using optical coherence tomography in migraine patients. $\mathrm{Br}$ J Ophthalmol. 2008:92(8):1069-75.

23. Sorkhabi R, Mostafaei S, Ahoor M, Talebi M. Evaluation of retinal nerve fiber layer thickness in migraine. Iran J of neurol. 2013;12(2):51.

24. Ao R, Wang R, Yang M, Wei S, Shi X, Yu S. Altered retinal nerve fiber layer thickness and choroid thickness in patients with migraine. Eur Neurol. 2018; 80(3-4):130-7.

25. Kirbas S, Tufekci A, Turkyilmaz K, Kirbas A, Oner V, Durmus M. Evaluation of the retinal changes in patients with chronic migraine. Acta Neurol Belg. 2013;113(2):167-72.

26. Gipponi S, Scaroni N, Venturelli E, Forbice E, Rao R, Liberini P, et al. Reduction in retinal nerve fiber layer thickness in migraine patients. Neurol Sci. 2013;34(6):841-5.

27. Gunes A, Demirci S, Tok L, Tok O, Demirci S, Kutluhan S. Is retinal nerve fiber layer thickness change related to headache lateralization in migraine? Korean J Ophthalmol. 2016;30(2):134-9.

28. Yülek F, Dirik EB, Eren Y, Simavl H, Uğurlu N, Çağıl N, et al. Macula and retinal nerve fiber layer in migraine patients: analysis by spectral-domain optic coherence tomography. Semin Ophthalmol. 2015;30(2):124-8.

29. Tunç A, Güngen BD, Evliyaoğlu F, Aras YG, Tekeşin AK. Evaluation of retinal nerve fiber layer, ganglion cell layer and macular changes in patients with migraine. Acta Neurol Belg. 2017;117(1):121-9.

30. Ekinci M, Ceylan E, Çağatay HH, Keleș S, Hüseyinoğlu N, Tanyıldız B, et al. Retinal nerve fibre layer, ganglion cell layer and choroid thinning in migraine with aura. BMC Ophthalmol. 2014;14(1):75.

31. Demircan S, Ataş M, Yüksel SA, Ulusoy MD, Yuvacı I, Arifoğlu HB, et al. The impact of migraine on posterior ocular structures. J Ophthalmol. 2015;2015: 868967.

32. Simsek IB. Retinal nerve fiber layer thickness of migraine patients with or without white matter lesions. Neuroophthalmology. 2017;41:7-11.

\section{Publisher's Note}

Springer Nature remains neutral with regard to jurisdictional claims in published maps and institutional affiliations.

\section{Submit your manuscript to a SpringerOpen ${ }^{\circ}$ journal and benefit from:}

- Convenient online submission

- Rigorous peer review

- Open access: articles freely available online

High visibility within the field

- Retaining the copyright to your article

Submit your next manuscript at $\boldsymbol{\nabla}$ springeropen.com 